\title{
Phonemer at WNUT-2020 Task 2: Sequence Classification Using COVID Twitter BERT and Bagging Ensemble Technique based on Plurality Voting
}

\author{
Anshul Wadhawan \\ Flipkart Private Limited \\ anshul. wadhwaneflipkart.com
}

\begin{abstract}
This paper presents the approach that we employed to tackle the EMNLP WNUT-2020 Shared Task 2 : Identification of informative COVID-19 English Tweets. The task is to develop a system that automatically identifies whether an English Tweet related to the novel coronavirus (COVID-19) is informative or not. We solve the task in three stages. The first stage involves pre-processing the dataset by filtering only relevant information. This is followed by experimenting with multiple deep learning models like CNNs, RNNs and Transformer based models. In the last stage, we propose an ensemble of the best model trained on different subsets of the provided dataset. Our final approach achieved an F1-score of 0.9037 and we were ranked sixth overall with F1-score as the evaluation criteria.
\end{abstract}

\section{Introduction}

Up till mid-June 2020, the coronavirus pandemic has caused $445 \mathrm{~K}$ deaths and has infected more than $8.2 \mathrm{M}$ people belonging to 215 regions and countries. This has led to a surge of panic and fear among people all around the globe. Recently, there has been rapid development in building monitoring systems (e.g. The Johns Hopkins Coronavirus Dashboard) to track any news regarding the outbreak, to let users know of any information related to the coronavirus for example, new cases emerging near the user's location. Most of the official sources from where the information is released are not updated very frequently and the information reported by such organizations may be stale, for example, WHO updates the information regarding the virus only once a day. These monitoring systems tend to use social network data like posts from Facebook or tweets from Twitter as an alternative source of information relating to the pandemic, generally by scraping relevant information or by crowd sourcing. However, due to increased panic and emotions among people, social media is flooded with massive amounts of data, e.g. about 4M COVID-19 English tweets are posted daily on twitter. But the major issue regarding this is that the majority of such tweets are uninformative and thus, do not impart useful information that can be used. Hence, any application that requires to update the news has to first filter these tweets to detect which among the tweets are actually useful. Manual approaches for this filtering task are not only cumbersome, frustrating and ineffective for vast amounts of data, but also costly. This calls for automated systems which can filter the information given huge amounts of mixed data, and thus serve as the motivation for the shared task. Although a lot of work has been done on the lines of sequence classification on general English texts (Agarwal et al., 2011; Bagheri and Islam, 2017), since the COVID-19 outbreak has grown in a short while, not many systems which deal with COVID-19 related texts have been developed.

In this paper, we describe our approach to tackle the WNUT 2020 shared task 2. The paper is structured as follows: Section 2 talks about the problem statement and provided dataset. Section 3 describes a step-by-step methodology process that we employ. Section 4 explains the experiments that were carried out along with a detailed discussion of the dataset, system settings and results of our experiments. Section 5 provides a brief conclusion of the paper along with the future scope of our research.

\section{Task Definition}

The WNUT-2020 Shared Task 2 (Nguyen et al., 2020) is based on a sequence classification problem wherein the aim is to identify whether an English Tweet related to the novel coronavirus (COVID-19) is informative or not. A tweet is said to be informative if it provides information regarding recov- 


\begin{tabular}{|c|c|c|c|}
\hline & Total & Positive & Negative \\
\hline Training set & 7000 & 3303 & 3697 \\
\hline Validation set & 1000 & 472 & 528 \\
\hline Test set & 2000 & 944 & 1056 \\
\hline
\end{tabular}

Table 1: Data Distribution

ered, suspected, confirmed, death cases, location or travel history of cases.

The goals of the shared task are:

1. To develop a language processing task that potentially impacts research and downstream applications.

2. To provide the community with a new dataset for identifying informative COVID-19 English Tweets.

To achieve the goals of the shared task, a dataset of 10K COVID-19 English tweets are provided, in which 4719 tweets are labelled INFORMATIVE and 5281 tweets are labelled UNINFORMATIVE. Each tweet is annotated by three independent annotators with an inter-annotator agreement score of Fleiss' Kappa at 0.818 . The $10 \mathrm{~K}$ dataset is divided into training/validation/test sets in the ratio $70 / 10 / 20$ with distribution as shown in table 1 .

Systems are evaluated using standard evaluation metrics, including accuracy, precision, recall and F1-score. However, the submissions are ranked by F1-score.

\section{Methodology}

We have split the proposed methodology in three steps- data preprocessing, deep learning models for sequence classification and ensemble process (bagging). The code corresponding to each of the steps has been made available online ${ }^{1}$ to facilitate further research.

\subsection{Data Pre-Processing}

The dataset provided in the WNUT shared task is not suitable to be processed directly by the models we plan to implement. This is due to the fact that the texts provided are tweets which are directly fetched from the website and users from all over the world have different ways of expressing their opinions. On manually going through the dataset, we find that the texts are very diverse in the sense

\footnotetext{
${ }^{1}$ https: / / github.com/anshulwadhawan/ BERT_for_sequence_classification_COVID
}

that many users post non-ascii characters such as emoticons, slang words for informal tweets, and spelling errors in words, etc. The dataset contains URLs replaced by the tag HTTPURL and user mentions replaced by the tag @USER. Apart from this, newline characters are also present within tweets. All the above discrepancies add to noise and do not contribute to being appropriate features for sequence classification.

In order to clean this data, we perform the following cleaning operations :

1. Remove all non-ascii characters i.e. characters belonging to the range $[\backslash x 00-\backslash x 7 f]$. We determined this range by parsing through the dataset and recording all non-ascii characters.

2. Remove all newline $(\backslash \mathrm{n})$ and tab $(\backslash \mathrm{t})$ characters.

3. Remove all HTTPURL and @USER tags.

\subsection{Deep Learning Models}

Deep learning techniques have recently shown great results in the domain of computer vision (Krizhevsky et al., 2012) and speech recognition (Graves et al., 2013). As far as natural language processing is concerned, most of the work involving deep learning makes use of word vector representations (Bengio et al., 2003; Yih et al., 2011; Mikolov et al., 2013) to carry out finer tasks like classification.

\subsubsection{CNNs}

(Kim, 2014) Convolutional Neural Networks are used to operate on local features with the help of convolving filters. CNNs have not only shown promising results in the domain of computer vision (Lecun et al., 1998), but they have also been utilized extensively for NLP tasks like search query retrieval (Shen et al., 2014), semantic parsing (Yih et al., 2014), sentence modeling (Kalchbrenner et al., 2014), and other traditional NLP tasks (Collobert et al., 2011).

\subsubsection{RNNs}

LSTM : LSTMs have shown great results in sequence classification problems like political sentiment classification (Rao and Spasojevic, 2016), by capturing the appropriate context. Also, they work towards solving the vanishing gradient problem (Hochreiter and Schmidhuber, 1997). BiLSTM : Bi-directional LSTMs have tackled a variety of sequence classification tasks (Wang et al., 2016) by 
considering the fact that context of a word depends on the words occurring before it as well as those occurring after it. Attention based BiLSTM : By including attention to a BiLSTM, we try to find out the specific words which have the greatest impact to the overall sentiment of the sequence under consideration.

\subsubsection{Transformer based models}

BERT (bert-base-cased): (Devlin et al., 2018) BERT is a bidirectional transformer based model pre-trained on a huge corpus of Wikipedia and Toronto Book Corpus which uses a combination of objectives meant for the tasks of next sentence prediction and masked language modeling. RoBERTa (roberta-base): (Liu et al., 2019) It is built on top of BERT by removing the next sentence prediction objective, changing key hyperparameters and training with increased learning rate values and batch sizes. ALBERT (albert-base-v2): (Lan et al., 2019) This is another variation of BERT which tries to increase the training speed of BERT and lower memory utilization by repeating layers which are split among groups and splitting the embedding matrix into two. XLNet (xlnet-base-cased): (Yang et al., 2019) This model extends over the Transformer-XL model by learning bidirectional contexts and maximizing the likelihood over different permutations of the input sequence factorization order after pre-training. XLM (xlm-mlmen-2048): (Lample and Conneau, 2019) This is a transformer based model with an option to choose the objective functions from the tasks of masked language modeling, casual language modeling, and translation language modeling. COVID Twitter BERT (ct-bert): (Müller et al., 2020) COVIDTwitter-BERT (CT-BERT) is a transformer-based model pre-trained on a corpus of $22.5 \mathrm{M}(633 \mathrm{M}$ tokens) COVID-19 related tweets.

\subsection{Ensemble Process - Bagging}

We merge the training and validation datasets provided in the task to create a global dataset. Then, we shuffle this global dataset and split it into training and validation datasets with the same ratio This process is repeated seven times to create seven sets of training and validation datasets, each of which have a random class distribution. The best performing model, based on validation scores on default training and validation split provided in the task, is trained from scratch on each of these seven sets of training and validation splits, and the

\begin{tabular}{|l|c|c|c|c|}
\hline \multirow{2}{*}{} & \multicolumn{2}{|c|}{ Train Set } & \multicolumn{2}{c|}{ Val Set } \\
\cline { 2 - 5 } & Pos & Neg & Pos & Neg \\
\hline Shuffle1 & 3285 & 3715 & 490 & 510 \\
\hline Shuffle2 & 3294 & 3706 & 481 & 519 \\
\hline Shuffle3 & 3305 & 3695 & 470 & 530 \\
\hline Shuffle4 & 3293 & 3707 & 482 & 518 \\
\hline Shuffle5 & 3313 & 3687 & 462 & 538 \\
\hline Shuffle6 & 3299 & 3701 & 476 & 524 \\
\hline Shuffle7 & 3293 & 3707 & 482 & 518 \\
\hline
\end{tabular}

Table 2: Data Distribution

predictions are recorded. Once we have the seven sets of predictions, we use a max-voting algorithm that is based on calculating mode of the seven predictions for each test instance to produce the final predictions.

\section{Experiments}

We experiment with ten deep learning models with the provided training and validation splits. Based on the scores produced above, we evaluate the final test predictions by training the best performing model with the seven synthesised randomly shuffled versions of the dataset, followed by merging the output predictions made on the test set. In this section, we present the dataset distribution, experimental settings, evaluation metrics, results and a brief analysis of the proposed system.

\subsection{Dataset}

The class-wise distribution in the training and validation splits of the provided as well as the shuffled datasets are shown in Table 2.

\subsection{System Settings}

For training the CNN, LSTM and BiLSTMs, word vectors for english language pre-trained on Common Crawl $\mathrm{l}^{2}$ and Wikipedia ${ }^{3}$ are downloaded ${ }^{4}$ and used using FastText ${ }^{5}$ library. These word vectors are used to create the embedding matrix which is further used for transforming the words of the input sentence. We use binary cross entropy loss function and adam optimizer for all the CNN and RNN models. All the layers except the last layer have relu activation function. Since the problem is a binary

\footnotetext{
${ }^{2}$ https: / / commoncrawl.org/

${ }^{3}$ https: / / www.wikipedia.org/

${ }^{4}$ https://dl.fbaipublicfiles.com/ fasttext/vectors-crawl/cc.en.300.bin.gz

${ }^{5}$ https://fasttext.cc/docs/en/ crawl-vectors.html
} 


\begin{tabular}{|l|c|c|c|c|}
\hline & $\mathrm{F} 1$ & $\mathrm{P}$ & $\mathrm{R}$ & $\mathrm{A}$ \\
\hline CNN & 0.787 & 0.805 & 0.771 & 0.804 \\
\hline LSTM & 0.807 & 0.850 & 0.769 & 0.827 \\
\hline BiLSTM & 0.822 & 0.806 & 0.838 & 0.829 \\
\hline Att-BiLSTM & 0.823 & 0.773 & 0.881 & 0.822 \\
\hline BERT & 0.891 & 0.875 & 0.908 & 0.896 \\
\hline RoBERTa & 0.899 & 0.886 & 0.913 & 0.904 \\
\hline ALBERT & 0.844 & 0.851 & 0.836 & 0.854 \\
\hline XLNet & 0.892 & 0.864 & 0.921 & 0.895 \\
\hline XLM & 0.870 & 0.850 & 0.891 & 0.875 \\
\hline CT-BERT & $\mathbf{0 . 9 1 4}$ & 0.869 & 0.963 & $\mathbf{0 . 9 1 5}$ \\
\hline
\end{tabular}

Table 3: Model Scores on Validation Set

classification, we use sigmoid activation function in the last layer. The training session is run for a total of 20 epochs and early stopping was inculcated in case of successive unproductive(in terms of f1-score) iterations. For all proposed RNNs, dropout of 0.2 and number_of_units of 150 are found to be the most effective. To fine-tune the transformer based models, we use pre-trained models, namely bert-base-cased, roberta-base, albertbase-v2, xlnet-base-cased and xlm-mlm-en-2048. We use hugging-face ${ }^{6}$ API to train all the transformer based models. We use a learning rate of $4 \mathrm{e}$ 5, epsilon_parameter_for_adam_optimizer of 1e-8, maximum_sequence_length of 128 and a batch_size of 8 due to hardware limitations. We train the models for 10 epochs and evaluate the model's performance on the validation set after every epoch.

\subsection{Results and Analysis}

The performance results of the proposed models on the given validation dataset in terms of $\mathrm{f1}$ score $(\mathrm{F} 1)$, precision $(\mathrm{P})$, recall $(\mathrm{R})$ and $\operatorname{accuracy}(\mathrm{A})$ have been presented in table 3 .

We can conclude the following from table 3 :

1. RNN based models perform better than CNN due to their context capturing potential.

2. Transformer based models perform better than both CNN and RNN based models due to more parallelization because of the fact that they don't need to traverse the input in order.

3. The COVID Twitter BERT (CT-BERT) outperforms all the other models by a significant margin. Higher recall is one major observation in this case. This is evident from the

\footnotetext{
${ }^{6}$ https://huggingface.co/transformers/
}

fact that the model is pre-trained on $22.5 \mathrm{M}$ COVID-19 related tweets.

From the above results, we choose CT-BERT to be the primary model to train on the seven randomly shuffled datasets as well as carry out inferences on the unseen dataset. The ensemble of produced predictions results in an F1-score of 0.9037 on the test dataset. The best performing model i.e. CT-BERT on being trained over the global dataset results in an F1-score of 0.8954 on the test dataset. This shows that by inculcating the bagging technique, a boost of $0.83 \%$ in F1-score is seen. This can be credited to the fact that by creating seven sets of randomly selected training examples, we essentially cover the entire global dataset. Although we exclude portions of the dataset by excluding randomly selected instances from the training dataset, we cover all the instances in the global dataset by selecting those labels which are predicted by majority of the seven trained CT-BERT models. This can be deduced from the assumption that a particular instance which is absent in the training set of one of the seven models is likely to be present in the training set of most of the remaining models.

\section{Conclusion and Future Work}

In this paper, we provide a detailed description of our approach to solve the EMNLP WNUT-2020 Shared Task 2. Our approach involves processing in three stages. In the first stage, we pre-process the provided dataset by cleaning and extracting only relevant information from the provided text. In the second stage, we experiment with several deep neural networks like CNN, RNNs and Transformer based networks like XLNet, XLM, BERT and its different variations. In the final phase, we introduce the idea of ensemble learning (bagging) to our solution which is a major improvement over the individual model. We submitted an ensemble and an individual system based on the CT-BERT model as our final entries to the shared task. The ensemble approach fetches us a private leaderboard rank of 6 with F1-score as the evaluation criteria. Our system promotes the usage of transformer based models pre-trained on relevant corpora and ensemble learning with as many candidate models as feasible. In future, we aim to explore the usage of non-ascii characters like emoticons as features for classification and imparting ensemble learning through an end-to-end deep learning solution. 


\section{References}

Apoorv Agarwal, Boyi Xie, Ilia Vovsha, Owen Rambow, and Rebecca Passonneau. 2011. Sentiment analysis of twitter data. In Proceedings of the Workshop on Languages in Social Media, LSM '11, page 30-38, USA. Association for Computational Linguistics.

Hamid Bagheri and Md Johirul Islam. 2017. Sentiment analysis of twitter data.

Yoshua Bengio, Réjean Ducharme, Pascal Vincent, and Christian Janvin. 2003. A neural probabilistic language model. J. Mach. Learn. Res., 3(null):1137-1155.

Ronan Collobert, Jason Weston, Leon Bottou, Michael Karlen, Koray Kavukcuoglu, and Pavel Kuksa. 2011. Natural language processing (almost) from scratch.

Jacob Devlin, Ming-Wei Chang, Kenton Lee, and Kristina Toutanova. 2018. Bert: Pre-training of deep bidirectional transformers for language understanding.

Alex Graves, Abdel rahman Mohamed, and Geoffrey Hinton. 2013. Speech recognition with deep recurrent neural networks.

Sepp Hochreiter and Jürgen Schmidhuber. 1997. Long short-term memory. Neural Comput., 9(8):1735-1780.

Nal Kalchbrenner, Edward Grefenstette, and Phil Blunsom. 2014. A convolutional neural network for modelling sentences. In Proceedings of the 52nd Annual Meeting of the Association for Computational Linguistics (Volume 1: Long Papers), pages 655665, Baltimore, Maryland. Association for Computational Linguistics.

Yoon Kim. 2014. Convolutional neural networks for sentence classification.

Alex Krizhevsky, Ilya Sutskever, and Geoffrey E. Hinton. 2012. Imagenet classification with deep convolutional neural networks. In Proceedings of the 25th International Conference on Neural Information Processing Systems - Volume 1, NIPS'12, page 1097-1105, Red Hook, NY, USA. Curran Associates Inc.

Guillaume Lample and Alexis Conneau. 2019. Crosslingual language model pretraining.

Zhenzhong Lan, Mingda Chen, Sebastian Goodman, Kevin Gimpel, Piyush Sharma, and Radu Soricut. 2019. Albert: A lite bert for self-supervised learning of language representations.

Y. Lecun, L. Bottou, Y. Bengio, and P. Haffner. 1998 Gradient-based learning applied to document recognition. Proceedings of the IEEE, 86(11):2278-2324.
Yinhan Liu, Myle Ott, Naman Goyal, Jingfei Du, Mandar Joshi, Danqi Chen, Omer Levy, Mike Lewis, Luke Zettlemoyer, and Veselin Stoyanov. 2019. Roberta: A robustly optimized bert pretraining approach.

Tomas Mikolov, Ilya Sutskever, Kai Chen, Greg Corrado, and Jeffrey Dean. 2013. Distributed representations of words and phrases and their compositionality. In Proceedings of the 26th International Conference on Neural Information Processing Systems - Volume 2, NIPS'13, page 3111-3119, Red Hook, NY, USA. Curran Associates Inc.

Martin Müller, Marcel Salathé, and Per E Kummervold. 2020. Covid-twitter-bert: A natural language processing model to analyse covid-19 content on twitter.

Dat Quoc Nguyen, Thanh Vu, Afshin Rahimi, Mai Hoang Dao, Linh The Nguyen, and Long Doan. 2020. WNUT-2020 Task 2: Identification of Informative COVID-19 English Tweets. In Proceedings of the 6th Workshop on Noisy User-generated Text.

Adithya Rao and Nemanja Spasojevic. 2016. Actionable and political text classification using word embeddings and lstm.

Yelong Shen, Xiaodong He, Jianfeng Gao, Li Deng, and Grégoire Mesnil. 2014. Learning semantic representations using convolutional neural networks for web search. In Proceedings of the 23rd International Conference on World Wide Web, WWW'14 Companion, page 373-374, New York, NY, USA. Association for Computing Machinery.

Yequan Wang, Minlie Huang, Xiaoyan Zhu, and Li Zhao. 2016. Attention-based LSTM for aspectlevel sentiment classification. In Proceedings of the 2016 Conference on Empirical Methods in Natural Language Processing, pages 606-615, Austin, Texas. Association for Computational Linguistics.

Zhilin Yang, Zihang Dai, Yiming Yang, Jaime Carbonell, Ruslan Salakhutdinov, and Quoc V. Le. 2019. Xlnet: Generalized autoregressive pretraining for language understanding.

Scott Wen-tau Yih, Xiaodong He, and Chris Meek. 2014. Semantic parsing for single-relation question answering. In Proceedings of ACL. Association for Computational Linguistics.

Wen-tau Yih, Kristina Toutanova, John C. Platt, and Christopher Meek. 2011. Learning discriminative projections for text similarity measures. In Proceedings of the Fifteenth Conference on Computational Natural Language Learning, pages 247-256, Portland, Oregon, USA. Association for Computational Linguistics. 